\title{
A synthetic RNA-mediated evolution system in yeast
}

Jensen, Emil D; Laloux, Marcos; Lehka, Beata J; Pedersen, Lasse E; Jakoinas, Tadas; Jensen, Michael K; Keasling, Jay D

Published in:

Nucleic Acids Research

Link to article, DOI:

10.1093/nar/gkab472

Publication date:

2021

Document Version

Publisher's PDF, also known as Version of record

Link back to DTU Orbit

Citation $(A P A)$ :

Jensen, E. D., Laloux, M., Lehka, B. J., Pedersen, L. E., Jakoinas, T., Jensen, M. K., \& Keasling, J. D. (2021). A synthetic RNA-mediated evolution system in yeast. Nucleic Acids Research, 49(15), [e88].

https://doi.org/10.1093/nar/gkab472

\section{General rights}

Copyright and moral rights for the publications made accessible in the public portal are retained by the authors and/or other copyright owners and it is a condition of accessing publications that users recognise and abide by the legal requirements associated with these rights.

- Users may download and print one copy of any publication from the public portal for the purpose of private study or research.

- You may not further distribute the material or use it for any profit-making activity or commercial gain

- You may freely distribute the URL identifying the publication in the public portal

If you believe that this document breaches copyright please contact us providing details, and we will remove access to the work immediately and investigate your claim. 


\title{
A synthetic RNA-mediated evolution system in yeast
}

\author{
Emil D. Jensen ${ }^{1}$, Marcos Laloux ${ }^{1}$, Beata J. Lehka ${ }^{1}$, Lasse E. Pedersen ${ }^{\oplus 1}$, \\ Tadas Jakočiūnas ${ }^{1}$, Michael K. Jensen ${ }^{\oplus 1,{ }^{\star}}$ and Jay D. Keasling ${ }^{1,2,3,4,5}$
}

\begin{abstract}
${ }^{1}$ Novo Nordisk Foundation Center for Biosustainability, Technical University of Denmark, Kgs. Lyngby, Denmark, 2Joint BioEnergy Institute, Emeryville, CA, USA, ${ }^{3}$ Biological Systems and Engineering Division, Lawrence Berkeley National Laboratory, Berkeley, CA, USA, ${ }^{4}$ Department of Chemical and Biomolecular Engineering \& Department of Bioengineering, University of California, Berkeley, CA, USA and ${ }^{5}$ Center for Synthetic Biochemistry, Institute for Synthetic Biology, Shenzhen Institutes of Advanced Technologies, Shenzhen, China
\end{abstract}

Received February 28, 2021; Revised April 13, 2021; Editorial Decision May 13, 2021; Accepted May 18, 2021

\begin{abstract}
Laboratory evolution is a powerful approach to search for genetic adaptations to new or improved phenotypes, yet either relies on labour-intensive human-guided iterative rounds of mutagenesis and selection, or prolonged adaptation regimes based on naturally evolving cell populations. Here we present CRISPR- and RNA-assisted in vivo directed evolution (CRAIDE) of genomic loci using evolving chimeric donor gRNAs continuously delivered from an errorprone T7 RNA polymerase, and directly introduced as RNA repair donors into genomic targets under either Cas9 or dCas9 guidance. We validate CRAIDE by evolving novel functional variants of an auxotrophic marker gene, and by conferring resistance to a toxic amino acid analogue in baker's yeast Saccharomyces cerevisiae with a mutation rate $>3,000$ fold higher compared to spontaneous native rate, thus enabling the first demonstrations of in vivo delivery and information transfer from long evolving RNA donor templates into genomic context without the use of in vitro supplied and pre-programmed repair donors.
\end{abstract}

\section{INTRODUCTION}

The ability to evolve biomolecules with tailor-made properties is inherently linked to mutagenesis, driving both natural and laboratory evolution. However, with the extreme high fidelity of genome replication, occurring with mutational frequencies in the order of one mutation per billion replicated DNA bases (i.e. $10^{-9}$ per base) (1), a multitude of directed evolution systems have been developed to increase both mutation rates and targeted mutation space $(2,3)$. While the vast majority of these systems rely on targeted mutagenesis of genomic loci using variant DNA donors designed and generated in vitro (4-7), a number of evolution systems have been developed to couple mutation and selection cycles in vivo in both bacteria $(2,8-11)$, yeast $(12-15)$, and mammalian cells (16). Such strategies circumvent the need for repeated cycles of human-guided design of mutational spectra, tedious hands-on genetic library construction, transformation, and selection, and have enabled targeted per-base substitution rates $>10000$-fold higher than those of host genomes (e.g. $10^{-5}-10^{-4}$ per base) $(14,17,18)$.

Importantly, when developing systems for directed evolution in vivo, orthogonal mutagenesis and subsequent targeted delivery of mutant donors is of primary importance, in order to efficiently dereplicate sequence to function under selective conditions $(19,20)$. To address these considerations, creative bioprospecting and mixing of biological parts from diverse hosts have proven successful, including delivery of DNA mutant donors by heterologous faulty DNA polymerases and targeted base-editing using proteinfusions strategies $(9-11,13,14,16)$. Interestingly, various viral phylogenies store genetic information with low replicative fidelity (up to $10^{-4}$ per base per infection) in the form of RNA-encoded genomes (21), and viral-derived components have been a rich source for prospecting parts for synthetic directed evolution systems $(8,10,22,23)$. Moreover, RNA has been shown to serve as direct templates for DNA double-strand break (DSB) repair by homologous recombination in vitro and in yeast, and later also in bacteria and human cell lines (7,24-27). Likewise, it has been demonstrated that RNA molecules synthesized in vivo can confer genome editing following induced DSBs $(28,29)$.

Based on this, RNA constitutes an interesting entry-point for development of directed evolution of DNA through RNA in vivo, yet this requires controlled delivery of diversified RNA donors to be established and means to target them to genomic loci of interest. Here we report the development of a synthetic in vivo directed evolution system for yeast using CRISPR/Cas9 or nuclease-deficient dCas9 (30-33) technology for RNA-programmed targeting of genomic loci with evolving chimeric donor gRNAs (cgRNAs) continuously delivered from an engineered low-fidelity T7

\footnotetext{
*To whom correspondence should be addressed. Tel: +45 6128 4850; Email: mije@biosustain.dtu.dk
} 
phage-derived RNA polymerase (T7RNAP). In this study, we first establish an inducible CRISPR/Cas9 system with cgRNA that allows for studying cgRNA-DNA repair in yeast. Next, we report the engineering and optimization of controlled and orthogonal delivery of multiple cgRNAs, and we demonstrate that the CRISPR- and RNA-assisted in vivo directed evolution (CRAIDE) system supports a mutation rate $>3000$-fold higher than native replication fidelity at user-defined genomic loci, thus providing the first example of an RNA-based directed evolution system in vivo.

\section{MATERIALS AND METHODS}

All strains, plasmids, oligos, gRNAs and gene blocks are listed in Supplementary Tables S2-6.

\section{Molecular cloning}

Oligonucleotides, gene block fragments, and doublestranded 90-mers were purchased from Integrated DNA Technologies (IDT). Fragments for USER cloning were amplified with Phusion U Hot Start PCR Master Mix from ThermoFisher Scientific (catalogue \#F533S), and assembly was done with USER enzyme (56) into $S f a \mathrm{AI} / \mathrm{Nb} . B s m \mathrm{I}-$ treated vectors as described previously (57). gRNA expression cassettes contain overhangs for cloning with universal USER-overhang oligos previously described (58). Oligo names in Supplementary Table S4 are indicative of their usage and are otherwise specifically mentioned in this section.

\section{gRNA plasmids}

Plasmid pEDJ8 expressing gRNAs for knock-out (KO) of RNH1 and RNH2O1 was constructed by assembling presynthesized gene blocks gEDJ1 and gEDJ2 into p0054 (59). Plasmid pEDJ332 was made by replacing the existing gRNA seed sequence in plasmid pCfB3050 (60) to target HIS3 (CAAGTGATTAACGTCCACAC) with oligos F-sgRNA_HIS3-KO and R-sgRNA_HIS3-KO.

\section{Enzymes}

Cas9 was cloned from p414-Tef1p-Cas9-CYC1t (Addgene \#43802) into vector pRS415U (61) with promoters from GAL1 or TEF1 to assemble pEDJ333 and pEDJ391, respectively. Plasmid pEDJ423 was made by inverse PCR first with oligos F-Cas9(D10A) and R-Cas9(D10A) on pEDJ391, and then with oligos F-Cas9(H840A) and R-Cas9(H840A) on the resulting plasmid. Wild-type T7RNAP was amplified from Escherichia coli (DE3) with oligos containing a truncated SV40 nuclear localization signal (NLS), and assembled with promoters from TEF1 or GAL1 into vector p0057 (59) to make pEDJ344 and pEDJ356, respectively. TEF1pro-T7RNAP was fused to GFP (amplified from gEDJ_GFP) in p0054 (pEDJ334) for expression analysis, and T7RNAP point mutants (pEDJ338-340, pEDJ342-343, pEDJ346, and pEDJ389) were generated by site-directed mutagenesis. epT7RNAP from pEDJ389 was then assembled with the GAL1 promoter into p0057 to make pMLB10.

\section{ADE2-targeting cgRNA designs}

Minimal vectors pEDJ400 and pEDJ437 consist of auxotrophic markers (URA3 or HIS3, respectively), ampicillin resistance gene $(\mathrm{AmpR})$, origin of replication for yeast $(2 \mu)$ and bacteria (pUC), and a USER cloning site. Oligos EDJ483-492 amplified fragments from pRS416U and genomic DNA for pEDJ400 assembly and for subsequently exchanging URA3 for HIS3 to make pEDJ437.

An $A D E 2$ disruption cassette, including the T7 promoter (gEDJ3), and gRNA scaffold fused to the T7 termination signal (tZ) (62) (gEDJ4) were assembled into p0054 (pEDJ350) or pEDJ400 (pEDJ399). pEDJ414 was made similarly by including ADH1t in the assembly. pEDJ372 was made by removing the $A D E 2$ disruption cassette from pEDJ350, and ADH1t was inserted into the EcoRI-SalI restriction sites upstream from the expression cassette in pEDJ350 to make plasmid ADH1t_pEDJ350. SUP4t was inserted into pEDJ350 in both orientations by inverse PCR to make plasmids sup4tF_pEDJ350 and sup4R_pEDJ350.

\section{HIS3-related cgRNA designs}

Antisense HIS3_AI expressed from the $\mathrm{T} 7$ promoter (gEDJ5) was assembled with ADH1t, gRNA:tZ, and $P G K 1$ promoter to constitute plasmid $\mathrm{pEDJ} 367$. T7 promoter was excluded ( $\mathrm{pEDJ} 368$ ) by inverse PCR, or gRNA scaffold was omitted from assembly (pEDJ370). pCfB2909 (60) contains an integration cassette for EasyClone site XII-5 (57) in yeast chromosomal DNA. Modified HIS3_AI $I^{\text {gen }}$ expressed from T7 promoter was synthesized as a gene block (gEDJ6) and assembled with ADH1t and $P G K 1$ promoter into pCfB2909 to make pEDJ375. Vector $\mathrm{pEDJ} 377$ was constructed by removing the $P G K 1$ promoter from pEDJ367 and modifying the seed sequence from TGTTAGTAAAAATTCGAGCT to TGTTAGTAAAAATTCCTCGA (change is underlined) by inverse PCR with F-pEDJ377_HIS3_AI(CTCGA) and R-pEDJ377_HIS3_AI (CTCGA) to match the artificial intron sequence residing in integrated pEDJ375.

pMLB2-4 for donor-size analysis were made by assembly into p0054 of $100 \mathrm{bp} / 150 \mathrm{bp} / 440 \mathrm{bp} \_H I S 3$ _AI(CTCGA) amplified from pEDJ377 with oligos MLB1-6, together with fragments ADH1t:T7pro and gRNA:tZ. For cgRNA expression analysis, pMLB7 was assembled by transferring ADH1t:T7pro:HIS3_AI(CTCGA):gRNA:tZ, amplified from pEDJ377 with F-ADH1t and R-sgRNAtZ, into pRS416U. pMLB8 was made by assembling HIS3_AI(CTCGA):gRNA:SUP4t, amplified from pEDJ377 with oligos MLB23 and MLB24, with ADH1t and SNR52pro into p0054.

pEDJ509-513 were made by inverse PCR on pEDJ437 containing the HIS3 genetic marker with oligos EDJ661 and EDJ662-666, respectively, to evaluate screened HIS3 mutants.

pMLB15 was made by ligation after inverse PCR on pEDJ375 with oligos EDJ610 and EDJ611, and plasmid pEDJ506 was made from inverse PCR on pMLB15 with oligos EDJ610 and EDJ654. pEDJ508 was made by ligation after PCR with EDJ657 and EDJ658 on pEDJ377, and the design was then transferred to pEDJ400 with oligos FADH1t-T7p and R-tZ. 


\section{CAN1 cgRNA design}

pEDJ465 was made by cloning an amplified fragment, comprising $660 \mathrm{nt}$ of the wild-type CAN1 sequence, with a ADH1t:T7pro fragment and sgRNA:tZ into vector pEDJ400 to express cgRNA against $C A N 1$.

\section{Baseline strain construction}

Strain CEN.PK2-1C was transformed with Cas9 (pEDJ391) to make baseline strain Sc35. Strain Sc36 was made by genetic deletion of RNH1 and RNH2O1 in Sc35 with $1 \mathrm{nmol}$ double-stranded 90-mer oligos and $1 \mu \mathrm{g}$ of gRNA plasmid pEDJ8. Native HIS3 was completely removed from Sc36 (Sc40) and Sc35 (Sc41) with $1 \mathrm{nmol}$ of double-stranded 90-mer oligo and $1 \mu \mathrm{g}$ gRNA plasmid pEDJ332. Strain Sc36 and Sc40 transformed with pEDJ391 were saved as Sc42 and Sc43, respectively. Sc43 was transformed with $3 \mu \mathrm{g}$ NotI-linearized pEDJ375 or pEDJ506 (HIS3 23 $\Delta 29$-XII-5) with $1 \mu \mathrm{g}$ gRNA plasmid pCfB3050 to make Sc71 and Sc138, respectively.

\section{ADE2 disruption analysis}

All media contains $2 \%$ glucose. CEN.PK2-1C was cotransformed with Cas9 (pEDJ391) or iCas9 (pCT; Addgene \#60620) and T7RNAP (pEDJ344) (Sc104 and Sc106, respectively), or an empty vector w/o T7RNAP (p0057) (Sc103 and Sc105, respectively). Sc103-106 were incubated $\mathrm{O} / \mathrm{N}$ in SC-LW, then diluted 10X and incubated for $4 \mathrm{~h}$ at $30^{\circ} \mathrm{C}$ with shaking prior to chemical transformation with relevant gRNA or cgRNA expression vectors. Sc103-104 were co-transformed with pEDJ372 and double-stranded 90-mer oligos with flanking homology to the $A D E 2$ break site. Transformed cells were resuspended in $100 \mu \mathrm{lmQ}$ water and transferred into $3 \mathrm{ml}$ liquid SC-LWU in a $15 \mathrm{ml}$ culture tube and incubation at $30^{\circ} \mathrm{C}$ for $72 \mathrm{~h}$ with shaking. Dilution series were plated after $72 \mathrm{hrs}$ on SC-LWU, and red/white ratios for $\sim 500$ colonies per plate were scored after 3 days of incubation at $30^{\circ} \mathrm{C}$. Chimeric red-white striped colonies $(<5 \%$ per plate) were not considered.

\section{Replica-plating analysis of HIS3 repair}

Incubations were performed at $30^{\circ} \mathrm{C}$. Sc 40 and Sc41 were transformed with galactose-inducible T7RNAP (pEDJ356), Cas9 (pEDJ333), and pEDJ367, pEDJ368, or pEDJ370 (Sc107-109 and Sc113-115 for Sc40 and Sc41, respectively). Control strains carried p0057 instead of pEDJ356 (Sc110-112 and Sc116-118 for Sc40 and Sc41, respectively). Transformed cells were plated on SC-LWU with $2 \%$ glucose. Isolated colonies were inoculated in 1 $\mathrm{ml}$ of SC-LWU with $2 \%$ glucose and grown $\mathrm{O} / \mathrm{N}$ with shaking. Dilution series were plated on the same media and incubated for 2 days prior to replica-plating. Replicaplating was done on $\mathrm{SC}-\mathrm{H}$ (to adjust for rare spontaneous conversions) and on SC-LWU with $2 \%$ glucose or $2 \%$ galactose. Plates were incubated for 48 hrs before transferring to $\mathrm{SC}-\mathrm{H}$, followed by 3 days incubation. Sc71 was co-transformed with pEDJ377, galactose inducible Cas9 (pEDJ333), and T7RNAP (pEDJ356) (Sc119) or p0057 (Sc120). The same replica-plate workflow as used above for scoring $\mathrm{His}^{+}$units was followed.

\section{Liquid induction analysis of HIS3 repair}

Incubations were performed at $30^{\circ} \mathrm{C}$ at $250 \mathrm{rpm}$. Strain Sc71 was transformed with pEDJ333 and pEDJ356, and then with pEDJ377, pMLB2, pMLB3, or pMLB4 (Sc139142, respectively) for donor-size analysis. Sc71 carrying pEDJ333 and pEDJ356 was transformed with pEDJ377, pMLB7, or pMLB8 (Sc143-145, respectively) for cgRNA expression analysis. $1 \mathrm{ml}$ of each saturated culture was pelleted, washed once in $500 \mu \mathrm{l}$ sterile $\mathrm{mQ}$ water, and resuspended in $200 \mu \mathrm{l}$ sterile $\mathrm{mQ}$ water that was transferred to $3 \mathrm{ml}$ of SC-LWU with $2 \%$ galactose $\left(\mathrm{OD}_{600} \sim 2.0\right)$ for 48 hrs induction. Cultures were adjusted to $\mathrm{OD}_{600}=2.0$, and $3 \times 1 \mathrm{ml}$ were plated on $\mathrm{SC}-\mathrm{H}$, and serial dilutions plated on SC-LWU with $2 \%$ glucose.

\section{Gain-of-function analysis of HIS3_23 $\Delta$ 29-XII-5 repair}

Incubations were performed at $30^{\circ} \mathrm{C}$ at $250 \mathrm{rpm}$. Sc146 and Sc147 were made by transforming Sc138 with pMLB10, pEDJ333, and pEDJ508 or pEDJ400 (ctrl), respectively. Isolated colonies were inoculated for each strain in $5 \mathrm{ml}$ of SC-LWU with $2 \%$ glucose for growth $\mathrm{O} / \mathrm{N}$. O/N cultures were washed once in sterile $\mathrm{mQ}$ water and adjusted to $\mathrm{OD}_{600}$ $\sim 2.0$ in $2 \mathrm{ml} \mathrm{SC}$-LWU with $2 \%$ galactose and incubated for $48 \mathrm{hrs}$. Final $\mathrm{OD}_{600}$ was determined before plating $300 \mu \mathrm{l}$ on five plates of SC-H and dilutions on SC-LWU both with $2 \%$ glucose for each replicate culture. Remaining culture for three biological replicates was added $5 \mathrm{ml}$ of SC-H with $2 \%$ glucose and additionally incubated for $72 \mathrm{~h}$. $500 \mu \mathrm{l}$ of saturated cultures was harvested by boiling with $400 \mathrm{mM}$ LiAce and $1 \%$ SDS for 10 min followed by ethanol precipitation and resuspended in $100 \mu \mathrm{lmQ}$ water. Amplicons were obtained by PCR with 2xOneTag master mix (ThermoFisher Scientific \#K01s71), oligos MLB26 and EDJ315 (genome), EDJ360 and EDJ353 (plasmid), and sequenced with the forward oligo for each reaction.

\section{CAN1 survival assay}

All media contained 2\% glucose. Sc36 was transformed with epT7RNAP (pEDJ389) and Cas9 (pEDJ391) or dCas9 (pEDJ423) to make Sc127 and Sc134. Sc127 and Sc134 were transformed with pEDJ400 or pEDJ465 to give Sc128 and Sc129 for Sc127, respectively, and Sc135 and Sc136 for Sc134, respectively. Auxotrophies in Sc36 were closed by co-transformation of pRS415 (LEU2), p0057 (TRP1), and pEDJ400 (URA3). Biological replicates were inoculated in $5 \mathrm{ml} \mathrm{SC}$-LWU and grown cultivated for $72 \mathrm{~h}$ in 15 $\mathrm{ml}$ culture tubes. Saturated cultures were plated on Delft supplemented with $20 \mathrm{mg}$ L-Histidine (Delft + ) and Delft+ supplemented with $60 \mu \mathrm{g} / \mathrm{ml}$ (in Figure 3) or $600 \mu \mathrm{g} / \mathrm{ml}$ (in Supplementary Figure S4) L-canavanine to apply selection for canl mutants. $50 \mu \mathrm{l}$ from three biological replicates $\pm \operatorname{cgRNA}$ were pelleted and supernatant discarded. Pellets were resuspended in water and plated, and the ratio of viable cells between strains expressing \pm cgRNA was determined after 3 days of incubation at $30^{\circ} \mathrm{C}$. Resulting genotypes from single colonies were determined by Sanger sequencing. Colony PCR was done with $2 x$ OneTag master mix and oligos F-CANI-Sanger and R-CAN1-Sanger to amplify endogenous $C A N 1$ for sequencing analysis. 


\section{Estimation of mutation frequencies and rate}

All data and calculations are presented in Supplementary Table S1. Mutational frequencies were obtained by scoring the number of resulting colonies on selective media following evolution. The average number of mutants was then divided by the number of viable cells per plated volume for each culture $(300 \mu \mathrm{l}$ for gain-of-function, $500 \mu \mathrm{l}$ for loss-of-function). Viable cells per volume were estimated from dilution series on non-selective media, and for gainof-function analysis, the number of generations during 48 hrs system induction was determined from $\Delta \mathrm{OD}_{600}$. Gainof-function mutation frequencies were divided by the number of underwent generations, and then by three to adjust for the space that allows for permissive mutations in the STOP codon (TAG) in HIS3 23 $\Delta 29$-XII-5 after repair with mutant $c g R N A_{-}$HIS3_stop (pEDJ508). Combined this estimates a mutation rate of $3.26 \times 10^{-6}$ per viable cell per generation per base. By comparison, commonly used $(17,22)$ online tools such as bz-rates $(63)$ and rSalvador (64) estimated comparable mutation rates of $2.80 \times 10^{-6}$ and $2.22 \times 10^{-6}$, respectively. Yet, as $b z$-rates and rSalvador assume neglectable low starting ODs, while CRAIDE requires starting $\mathrm{OD}_{600} \sim 2.0$, we consider the mutation rate of $3.26 \times 10^{-6}$ per viable cell per generation per base most accurate.

\section{Flow cytometry analysis}

Strains Sc121-126 and p0054 were diluted 1:10 from O/N cultures into fresh $500 \mu \mathrm{l} \mathrm{SC}-\mathrm{U}$ and incubated at $30^{\circ} \mathrm{C}$ with shaking for $24 \mathrm{~h}$ prior to analysis. Cultures were diluted 1:5 in $150 \mu 1$ with Phosphate Buffer Saline (PBS) from Life Technologies immediately before analysis by flow cytometry on the BD LSR Fortessa X-20 (BD Biosciences). Blue laser at $488 \mathrm{~nm}$ was used to analyse 10,000 single cells for each population, and FlowJo software (TreeStar Inc.) was used to process data and to calculate arithmetic mean fluorescence intensity values.

\section{Statistical analysis}

Significance was determined by two-tailed Student's t-test using at least three biological or technical replicates.

\section{Media}

One liter of mineral media (Delft) with $2 \%$ glucose (65) con-

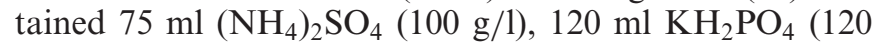
$\mathrm{g} / 1), 10 \mathrm{ml} \mathrm{MgSO} 4,7 \mathrm{H}_{2} \mathrm{O}(50 \mathrm{~g} / \mathrm{l}), 2 \mathrm{ml}$ trace metals, $1 \mathrm{ml}$ vitamins, and $20 \mathrm{~g}$ glucose. One liter of trace metals contain $4.5 \mathrm{~g} \mathrm{CaCl}_{2} \cdot 2 \mathrm{H}_{2} \mathrm{O}, 4.5 \mathrm{~g} \mathrm{ZnSO}_{4} \cdot 7 \mathrm{H}_{2} \mathrm{O}, 3 \mathrm{~g} \mathrm{FeSO} \cdot 7 \mathrm{H}_{2} \mathrm{O}$, $1 \mathrm{~g} \mathrm{H}_{3} \mathrm{BO}_{3}, 1 \mathrm{~g} \mathrm{MnCl}_{2} \cdot 4 \mathrm{H}_{2} \mathrm{O}, 0.4 \mathrm{~g} \mathrm{Na}_{2} \mathrm{MoO}_{4} \cdot 2 \mathrm{H}_{2} \mathrm{O}, 0.3$ $\mathrm{g} \mathrm{CoCl} 2 \cdot 6 \mathrm{H}_{2} \mathrm{O}, 0.1 \mathrm{~g} \mathrm{CuSO}_{4} \cdot 5 \mathrm{H}_{2} \mathrm{O}, 0.1 \mathrm{~g} \mathrm{KI}$, and $15 \mathrm{~g}$ EDTA. One liter of vitamins contain $50 \mathrm{mg}$ biotin, $200 \mathrm{mg}$ p-aminobenzoic acid, $1 \mathrm{~g}$ nicotinic acid, $1 \mathrm{~g}$ Ca-pantotenate, $1 \mathrm{~g}$ pyridoxine $\mathrm{HCl}, 1 \mathrm{~g}$ thiamine $\mathrm{HCl}$, and $25 \mathrm{~g}$ myoInositol. Synthetic complete dropout media were bought from Sigma-Aldrich.

\section{RESULTS}

\section{Engineering orthogonal cgRNA delivery in yeast}

In order to develop a targeted in vivo evolution system, we initially sought to combine elements of RNA-programmed genome targetability of CRISPR/Cas9, and error-prone RNA polymerase for expression of donor-coupled chimeric gRNAs (cgRNAs), serving as repair templates at targeted genomic loci $(31,32,34)$. For choice of RNA polymerase, we selected bacteriophage T7RNAP, originally reported to produce mRNA transcripts, and more recently also functional gRNAs, in yeast $(35,36)$. Importantly, beyond orthogonal transcription relying on the high $\mathrm{T} 7$ promoterspecificity and synthesis of untranslated RNA in yeast by T7RNAP (37), transcriptional mutagenesis can be adjusted by evolved T7RNAPs with nucleotide substitution error rates up to $1.25 \times 10^{-3}$ demonstrated in vitro and in E. coli (38), making T7RNAP of particular interest for in vivo evolution.

From this design, we first evaluated genome editing efficiency at the $A D E 2$ locus using wild-type T7RNAP in combination with Streptococcus pyogenes Cas9, and an $A D E 2$ gRNA (Figure 1A, Supplementary Figure S1). When co-transforming a 90-mer double-stranded DNA oligo (dsOligo) to knock-out $A D E 2$ we observed modest $2 \%$ genome editing efficiency, whereas leaving out dsOligo lowered efficiency to $0.04 \%$, while no $A D E 2$ disruption was observed when both T7RNAP and dsOligo were omitted (Figure 1A, Supplementary Figure S1). To investigate in vivo delivery of RNA-mediated repair templates we next constructed chimeric donor gRNA (cgRNA) based on a 200 nucleotide $5^{\prime}$-primed extension of gRNA homologous to $A D E 2$ with PAM site and four PAMproximal seed bases omitted to safeguard target site from repetitive cutting and frameshift-induced knock-out following cgRNA-templated DSB repair, respectively (Figure 1B, Supplementary Figure S1). We also tested a Cas9 variant reported to have improved genome editing efficiency (iCas9: Cas9 ${ }^{\mathrm{D} 147 \mathrm{Y}, \mathrm{P} 411 \mathrm{~T}}$ ) (39). Indeed, from cotransformations of T7RNAP and cgRNA together with either iCas9 or Cas9, we obtained $86 \%$ and $6 \%$ gene editing, respectively (Figure 1B). However, in both cases, background gene editing efficiencies when T7RNAP was omitted reached $43 \%$ and $3 \%$, indicating leaky cgRNA expression from the first-generation plasmid design (pEDJ350; Figure 1B).

As orthogonal and controlled delivery of evolving cgRNAs by T7RNAP is of paramount importance for practical applications, we mitigated high background gene editing by (i) removing unannotated sequences in the cgRNA expression plasmid targeting ADE2 (pEDJ399; Figure 1B), and (ii) introducing Pol II RNAP terminator from $A D H 1$ gene (ADH1t) upstream the T7 promoter on the pEDJ399 plasmid (pEDJ414; Figure 1B). From these two approaches, background gene editing was lowered to $17 \%$ and $2 \%$ for iCas9, and to $1 \%$ and $0.2 \%$ for Cas9 (Figure 1B), while at the same time maintaining T7RNAP-mediated gene editing efficiencies of 73-79\% and 7-9\% for iCas9 and Cas9, respectively. Moreover, inserting Pol III RNAP terminator SUP4 (SUP4t) upstream of the T7 promoter in pEDJ350 did not 
A

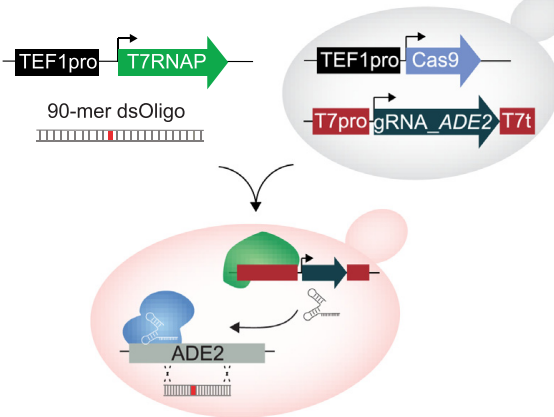

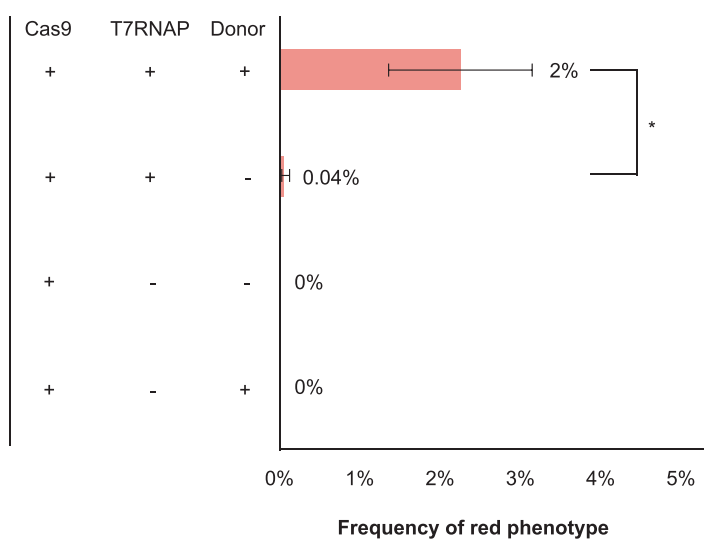

B

200 bp ADE2 disruption donor (ADE2d)
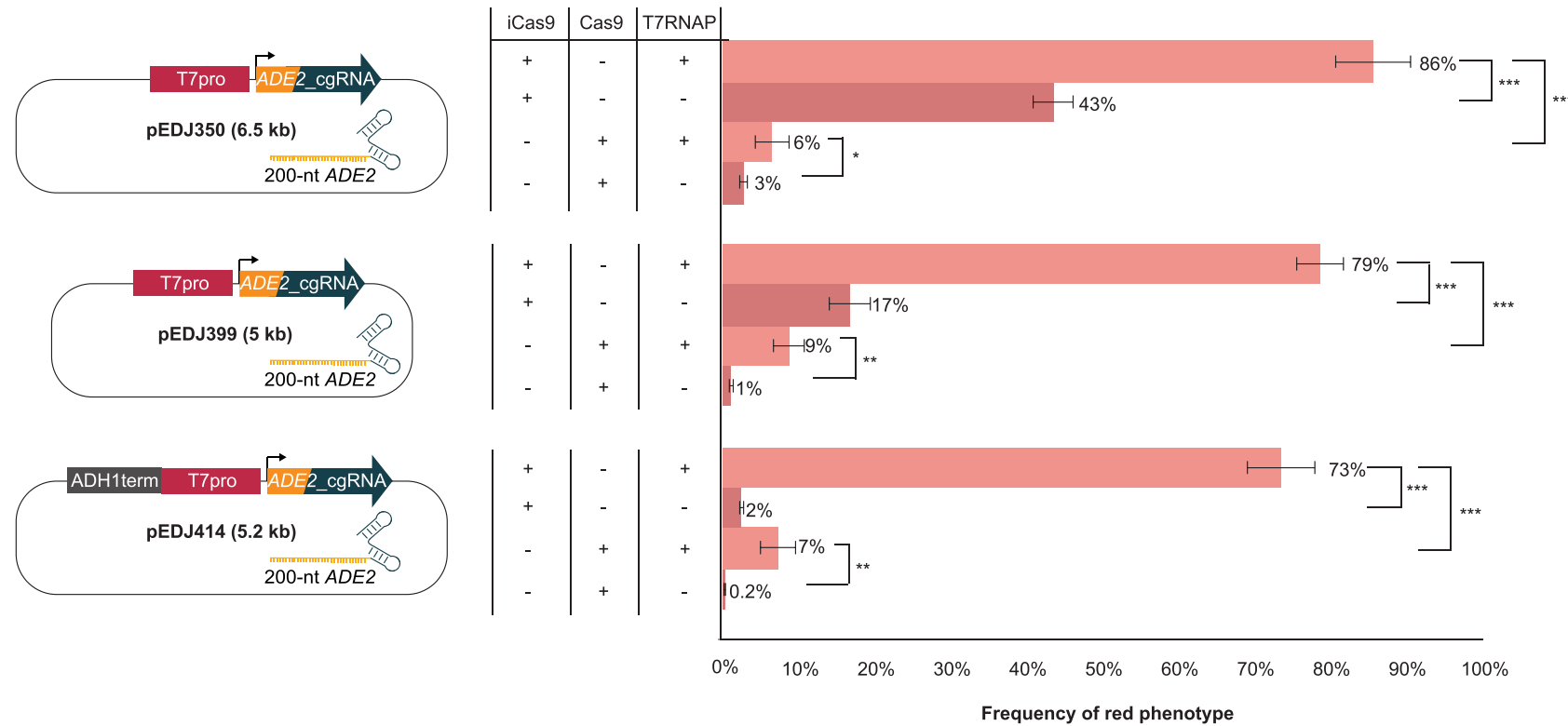

Figure 1. T7 RNA polymerase controls functional expression of gRNA and chimeric gRNAs (cgRNAs) in yeast. (A) Schematic illustration of experimental set-up, and frequencies of red colonies as a proxy for ade 2 knock-out and adenine deficiency. T7 RNA polymerase (T7RNAP) is indispensable for red colony formation in yeast cells expressing Cas9 under the control of the constitutive TEF1 promoter (TEF1pro) and gRNA under the control of the T7 promoter (T7pro), when co-transformed with a linear double-stranded oligo (dsOligo) targeting disruption of $A D E 2$. Mean frequencies of red colony formation \pm S.D. from three $(n=3)$ biological replicate experiments. (B) Frequencies of red colonies in yeast cells expressing chimeric gRNA (cgRNA) targeting $A D E 2$ ( $A D E 2$ _cgRNA). The cgRNA is based on a 200 nucleotide 5'-primed extension of gRNA homologous to ADE2 with PAM site and four PAM-proximal seed bases deleted (disruption donor; ADE2d). Frequencies from yeast cells expressing either improved Cas9 (iCas9) or Cas9 in the presence or absence of T7RNAP are shown as mean \pm S.D. from three $(n=3)$ biological replicate experiments.

affect background expression, whereas inserting ADH1t in pEDJ350 reduced background expression similarly to the reduction observed in pEDJ414, indicating that the insulating properties of the ADH1t are indispensable for tight control of cgRNA expression (Supplementary Figure S2).

In summary, we established an adjustable genome engineering system based on Cas9 variants and orthogonal delivery of functional cgRNA.

\section{Repair of plasmid DNA by cgRNA}

To demonstrate that DSBs are repaired by T7RNAPmediated delivery of cgRNA, and not by DNA-DNA homologous recombination between the cgRNA-expressing plasmid and the genomic target locus, we leveraged a previously established system to study transcript-mediated DSB repair (40). In this system, spliced antisense HIS3 transcripts can serve as homologous templates to repair DSBs 
in the HIS3 ORF interspersed by an artificial intron $(A I)$, and subsequently allow for conditional expression of native HIS3 transcripts read in sense orientation (Figure 2A) $(40,41)$. In our modified system we initially fused cgRNA $3^{\prime}$-end of antisense HIS3 (HIS3_AI_cgRNA) expressed under the control of the T7 promoter and introduced this plasmid into cells with T7RNAP and Cas9 expression induced by galactose. We used this design to test if CRISPR/Cas9mediated DSB in the plasmid could be repaired by spliced HIS3_AI_cgRNA transcripts originating from the plasmid itself (cis). An early committed step for RNA-mediated repair of DSB is the formation of RNA-DNA duplexes, and RNase activity has been shown to inhibit RNA-DNA repair in eukaryotes $(40,42)$. For this reason we tested T7RNAP-mediated delivery of HIS3_AI_cgRNA in both wild-type cells and in cells deleted for RNase H1 (RNHI) and RNase H2 (RNH201) (40). Using replica-plate workflows we grew up wild-type and rnhl rnh201 cells with glucose, then replicated colonies onto galactose or glucose, and finally onto selective media without histidine to score colony-forming units following 3 days cultivation (Figure 2A). When inducing expression of T7RNAP and Cas9 in rnhl rnh201 cells, $36 \%$ of the colonies turned histidine prototrophic (pEDJ367; + galactose), whereas only $0.1 \%$ of colonies from glucose control medium survived without supplemented histidine (Figure 2B). Furthermore, from galactose-induction medium, the number of histidine prototrophic colonies drastically decreased to $0.2 \%$ and $3 \%$ from cells with deletions of either T7 promoter or $\operatorname{cgRNA}$ in the HIS3_AI_cgRNA expressing plasmid, respectively, and no colonies appeared without induction (Figure 2B). Finally, we never detected any colonies on selective medium following induction of Cas9 and T7RNAP in wild-type cells, and neither did we observe any colonies from cells without T7RNAP (Figure 2B).

Taken together, these results highlight a tightly controlled cgRNA delivery system for transcript-mediated repair in RNase-deficient yeast.

\section{Repair of genomic DNA by cgRNA}

Next, to enable a portable evolution system for delivery of candidate cgRNAs to target genomic loci, we determined if plasmid-based cgRNA expression could also support genome editing (trans). To enable this analysis, we changed 5 PAM-proximal bases (GAGTC) in the original cgRNA of the HIS3_AI_cgRNA plasmid into complementary bases (CTCGA, HIS3_AIgen ), to specifically allow Cas9 to be guided to an integrated new synthetic $H I S 3 \_A I$ design matching seed sequence CTCGA only found in the genomic target locus (Figure 2C). Repeating the workflow described above, induction of Cas 9 and T7RNAP in rnh1 rnh201 cells supported increased colony numbers $(7 \%)$ under selective conditions, whereas control cells without T7RNAP expression only supported modest colony numbers $(0.2 \%)$ under the same conditions, confirming that T7RNAP mediated expression of cgRNA directs Cas9 and templates DSB repair in genomic contexts (Figure 2C).

To further test how cgRNA expression influences cgRNA-DNA repair, we induced cells in liquid dropout media and compared cgRNA expression from multicopy plas- mids $(2 \mu)$ and centromeric plasmids (CEN/ARS). Here, we found that using multicopy plasmids for cgRNA expression was $>4$-fold $(P<0.005)$ more efficient than expression from centromeric plasmids (Figure 2D), whereas the use of more active native RNA polymerase III SNR52 promoter (36) to drive cgRNA expression did not further improve cgRNA-DNA repair (Figure 2D). This result indicates that cgRNA expression is not a limitation when expressed with T7RNAP from multicopy plasmids, and furthermore serves to illustrate that the cgRNADNA repair system can be scored based on simple liquid passaging.

Moreover, since homology size is paramount to efficient DNA-DNA repair (43), and has also been demonstrated to affect RNA-DNA repair (24), we next investigated cgRNADNA repair efficiencies of differently sized truncations of the cgRNA donor sequence compared to full-length donors $(670 \mathrm{nt})$. Here, we found that longer homology regions $(670$ nt) were $\sim 86$-fold more efficient for cgRNA-DNA repair compared to cgRNAs with short homology donors of 100 nt (Figure 2E).

In summary, controllable plasmid-based cgRNA expression on plates or in liquid cultures can be designed to target the genome, where expression of long cgRNA donors from $2 \mu$ plasmids improves cgRNA-DNA repair efficiency.

\section{cgRNA-mediated directed evolution in vivo}

Next, to investigate if DSB can be repaired by erroneous cgRNA donors, and thereby make way for establishment of RNA-mediated directed evolution in genomic contexts, we combined our established system for control of cgRNA delivery and Cas9-mediated targeting with the expression of a recently described error-prone T7RNAP double mutant (T7RNAP ${ }^{\mathrm{F} 1 \mathrm{~L} / \mathrm{T} 613 \mathrm{~A}}$ ) (22). T7RNAP ${ }^{\mathrm{F} 11 \mathrm{~L} / \mathrm{T} 613 \mathrm{~A}}$ was originally derived from a triple mutant with error-rates reported in $E$. coli studies to approximate $1.25 \times 10^{-3}$ per transcribed base (38). However, though the triple-mutant did not express well in yeast, T7RNAP ${ }^{\mathrm{F} 11 \mathrm{~L} / \mathrm{T} 613 \mathrm{~A}}$ was observed to increase $A D E 2$ disruption over T7RNAP by 5 -fold (Supplementary Figure S3), and was therefore sought for evolving cgRNAs and genomic loci in vivo.

To test RNA-mediated directed evolution using T7RNAPF11L/T613A, we initially targeted resistance towards the toxic arginine analogue, L-canavanine, as a proxy for genome evolution (36), by directing Cas9 to genomic CAN1 using evolving 660-nt cgRNA donors (Figure 3A). Following three days of directed evolution in liquid cultures, we scored mutation frequency based on canavanine-resistance observed in Cas9- and T7RNAP ${ }^{\mathrm{F} 11 \mathrm{~L} / \mathrm{T} 613 \mathrm{~A}}$-expressing cells either with or without the expression of CAN1_cgRNA. Here, we identified mutation frequencies of $1.8 \times 10^{-5} \pm 1.3$ $\times 10^{-6}$ and $3 \times 10^{-2} \pm 6 \times 10^{-4}$ for cells without and with cgRNA expressed, respectively, totalling 1653 -fold higher mutation frequencies in cgRNA-expressing populations compared to populations not expressing cgRNA $(P=$ $1.14 \mathrm{E}-07$ ) (Figure 3B). Sequencing of the genomic $C A N 1$ locus identified $\mathrm{K} 405 \mathrm{~N}$ and S442Stop mutations in strains expressing T7RNAPF11L/T613A with cgRNA and Cas9, with mutational spectrum spanning up to 107 bases from the DSB (Figure 3C). None of the few colonies arising 

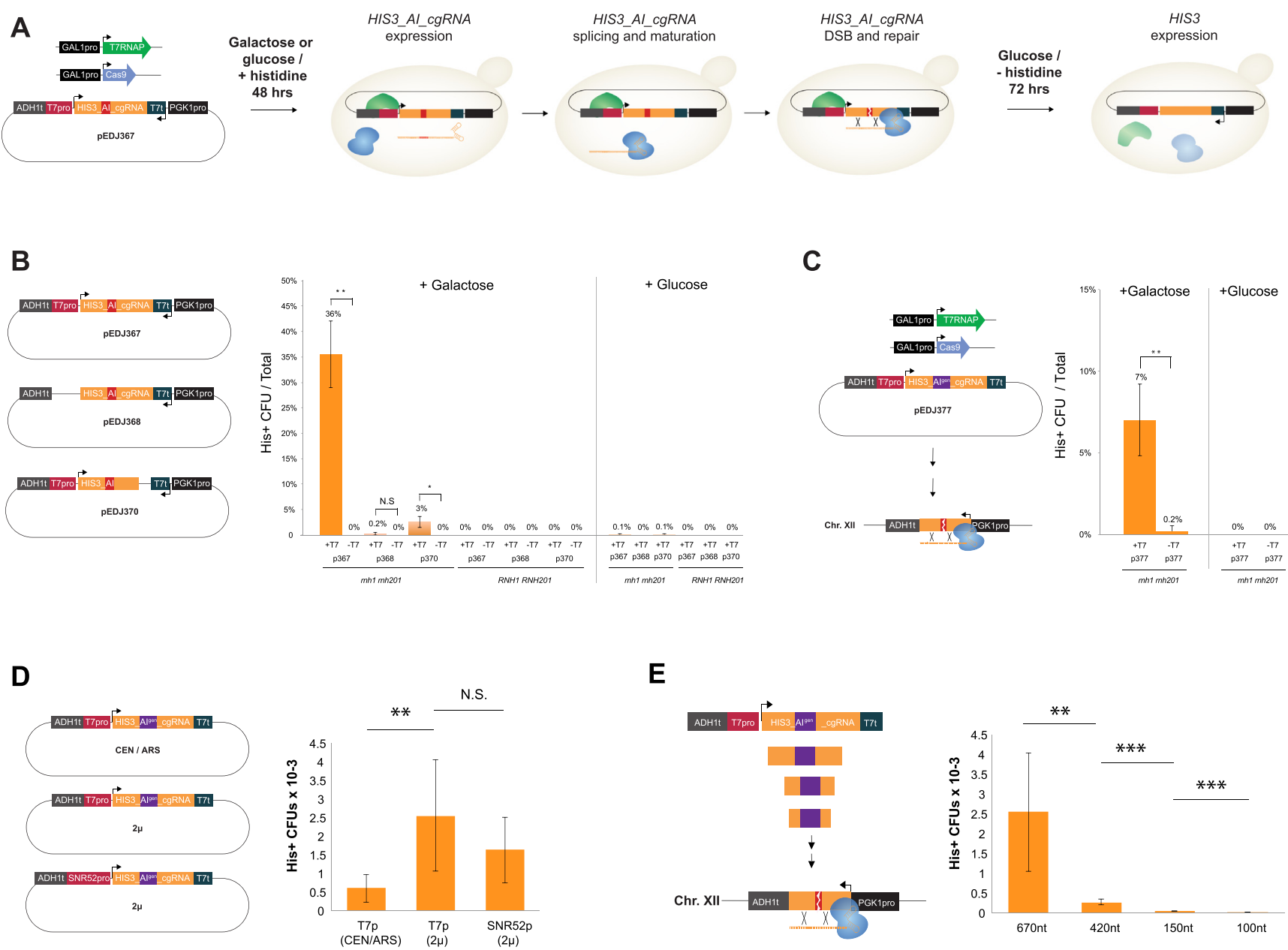

Figure 2. Cas9-mediated DNA double-strand breaks are repaired by RNA donors encoded in chimeric guide RNAs. (A) Schematic outline of the inducible replica-plating work-flow used for inferring repair of DNA double-strand breaks by donor RNA encoded in cgRNAs. (B) Dual-expression $2 \mu$ plasmidbased designs co-transformed into yeast together with Cas9 alone or with both T7RNAP and Cas9, show that inducible expression of T7RNAP enables efficient repair of Cas9-mediated DSB in the plasmid-encoded ( cis) artificial intron (AI) positioned in the HIS3 open reading frame when expressed in RNase-deficient (rnhl rnh201) yeast. $\mathrm{His}^{+}$colony forming units (CFUs) out of total colonies are shown. (C) Cas9-mediated DSB of HIS3_AIgen in a single-copy genome-encoded (trans) his3 AI-disrupted reading frame (Sc71) can be repaired by donor RNA encoded in cgRNAs expressed by inducible T7RNAP in RNase-deficient ( $r n h 1$ rnh201) yeast. (D) cgRNA expression impacts cgRNA-DNA repair efficiency. A liquid assay was conducted with $r n h 1$ rnh201 strains with the cgRNA construct from pEDJ377 contained in centromeric (CEN/ARS) or $2 \mu$ plasmids and expressed from T7 promoter (T7pro) or SNR52 promoter (SNR52pro) as indicated. Genome-integrated HIS3_AI gen was the target, and T7RNAP and Cas9 were inducibly expressed with galactose for $48 \mathrm{~h}$ prior to plating and $\mathrm{His}^{+}$scoring. Colony-forming units (CFUs) were calculated relative to plating efficiency on non-selective media. (E) cgRNA-DNA repair with various donor sizes were investigated as in (D) by symmetric truncations of the cgRNA construct contained in pEDJ377 targeting genome-integrated HIS3_AI ${ }^{g e n}$. For (B-E) frequencies of histidine prototrophic colonies and their error bars are shown as mean \pm S.D. from three $(n=3)$ biological replicate experiments and significance determined from Student's t-test, where $* P<0.05$, $* * P<0.005$, $* * * P<0.0005$, and N.S. $=$ not significant.

from strains lacking cgRNA had CAN1 mutations within the donor region (Figure 3C).

Encouraged by these results, and by the fact that mutagenesis associated with nuclease-deficient Cas9 (dCas9) (44) has been observed previously (45-47), we next sought to test if dCas9 could facilitate RNA-DNA editing without Cas9induced DSB using the CANI RNA-DNA repair screen, and 10X higher concentrations of L-Canavanine compared to Figure 3 to diminish residual growth (Supplementary Figure S4A and S4B). Here, Cas9 performed 3.5-fold better than $\mathrm{dCas} 9(P=0.017)$ with resistant colonies appearing at a frequency of $2.2 \times 10^{-5}$ and $6.3 \times 10^{-6}$ in viable cells, respectively, after induction (Supplementary Figure S4C), while dCas9 sustained higher cell densities $(P=0.031)$. By contrast, strains with no cgRNA expression appeared 229fold less frequently on selective plates compared to when both Cas 9 and cgRNA were expressed $\left(9.6 \times 10^{-8} ; P=\right.$ 0.0045 and $P=0.011$ for Cas9 and dCas9, respectively; Supplementary Figure S4C). These results provide a first demonstration of using dCas9 for cgRNA-DNA editing.

Finally, to fully demonstrate the applicability of directed evolution with cgRNA-DNA repair, we tested CRAIDE for gain-of-function mutagenesis in a genomic locus. For this purpose, we targeted a genome integrated design (HIS3_23 $429-X I I-5)$ lacking 29 bases of the HIS3 open reading frame, and hence rendering cells unable to 




3. cgRNA-gDNA recombination at CAN1 locus

C

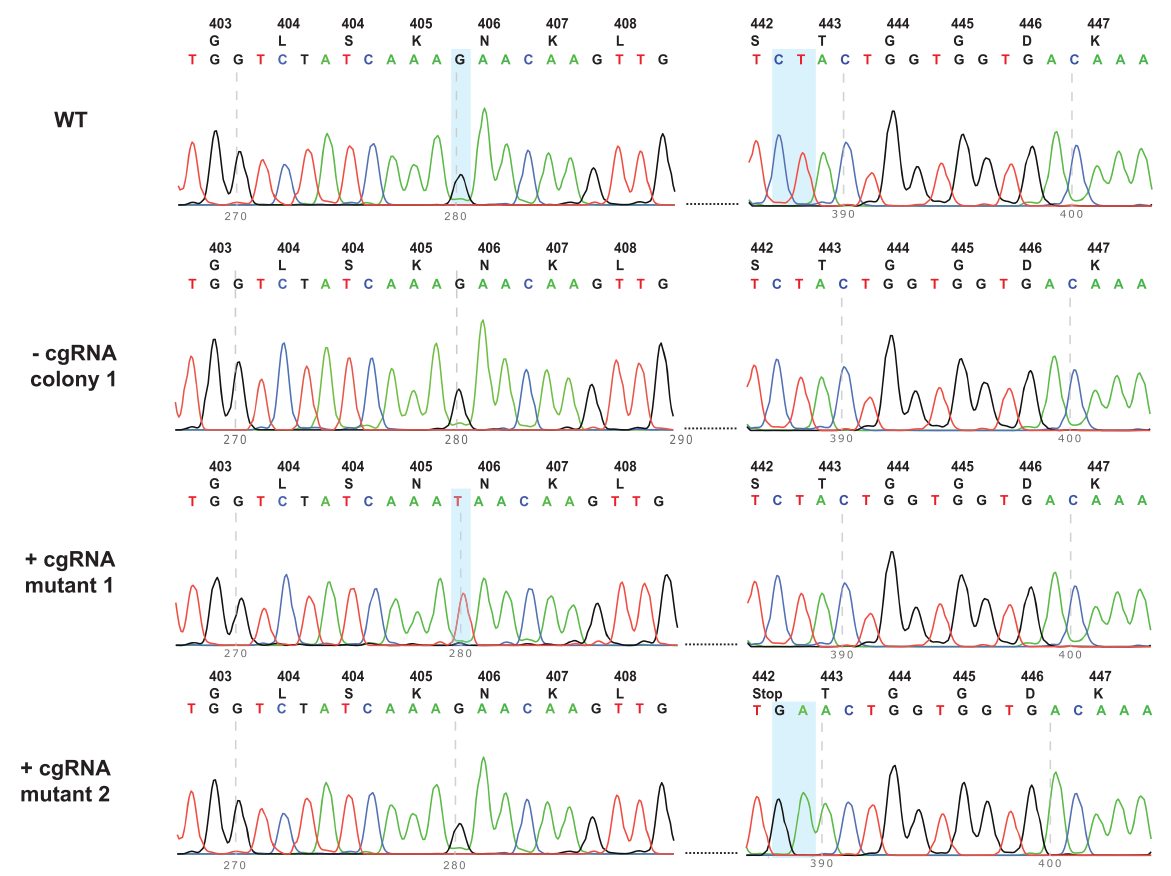

Figure 3. Orthogonal delivery of cgRNAs for targeted genome evolution in vivo. (A) Schematic outline of CRISPR-assisted programing of RNA-mediated in vivo directed evolution (CRAIDE). (B) Evolving resistance to L-canavanine by error-prone T7RNAP ${ }^{\mathrm{F} 11 \mathrm{~L} / \mathrm{T} 613 \mathrm{~A}}$-mediated transcription of cgRNAs encoding a 660-nt donor for positive selection of CAN1 disruption. Representative colony numbers on selective (+CAN) and non-selective ( - CAN) plates. Following 3 days of evolution in liquid cultures, $50 \mu \mathrm{l}$ of control (-cgRNA) and CRAIDE ( + cgRNA) cultures were plated onto selective and non-selective plates, and mutation frequency scored based on numbers of L-canavanine-resistant colonies on three $(n=3)$ biological replicates. For each plate $50 \mu 1$ of saturated liquid culture was plated. For - CAN plates, the $50 \mu 1$ was diluted $500 \times$ before plating. (C) Sanger-based sequencing of genomic CAN1 locus in WT cells and two canavanine-resistant (canl_mut.1 and can1_mut. 2) colonies from CAN1-cgRNA expressing CRAIDE cultures.

grow without histidine supplementation. Here, galactose inducible T7RNAP $\mathrm{F}^{\mathrm{F} 1 \mathrm{~L} / \mathrm{T} 613 \mathrm{~A}}$ and Cas9 were expressed together with $c g R N A_{-} H I S 3 \_s t o p$ containing a STOP codon at HIS3 position K71 (pEDJ508), which is surrounded by the $29 \mathrm{bp}$ deletion in the genomic design to rule out the possibility of NHEJ repair in surviving mutants. More specifically, the $c g R N A \_H I S 3 \_s t o p$ was engineered to contain a STOP codon (A211T; AAG $\rightarrow$ TAG) three bases upstream from the artificial intron and ten bases from the Cas9generated DSB in HIS3 23 $429-X I I-5$ (Figure 4A). Hence, by design, only induced cells successfully repaired with a
cgRNA which had the encoded STOP codon evolved into a permissive mutation would be able to sustain growth under selection (w/o histidine supplementation). Repeating the liquid passaging set-up as previously adopted (see Figure 2D and E) we induced seventeen replicate cultures each transformed with the plasmid expressing $c g R N A_{-} H I S 3 \_s t o p$ (pEDJ508), along six replicate control cultures transformed with empty no-cgRNA vector (pEDJ400), for $48 \mathrm{hrs}$ under non-selective conditions (galactose, with histidine; Figure 4B). Next, cultures were plated on histidine dropout media to score the mutation frequency, and for a subset also 
A

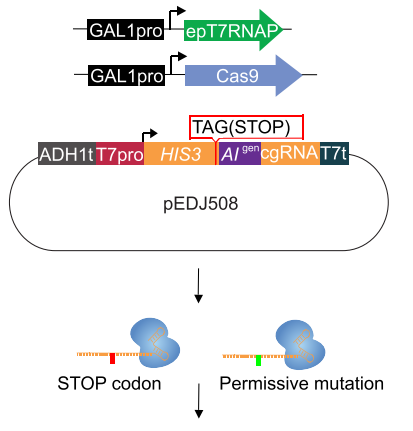

HIS3_23A29-XII-5

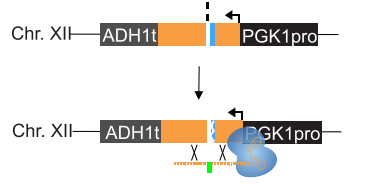

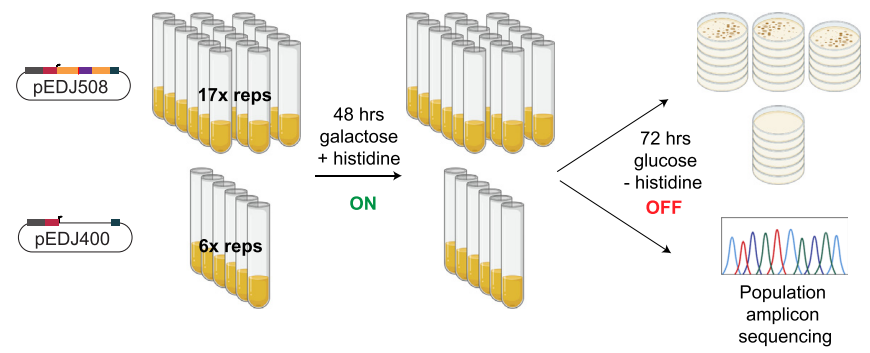

C

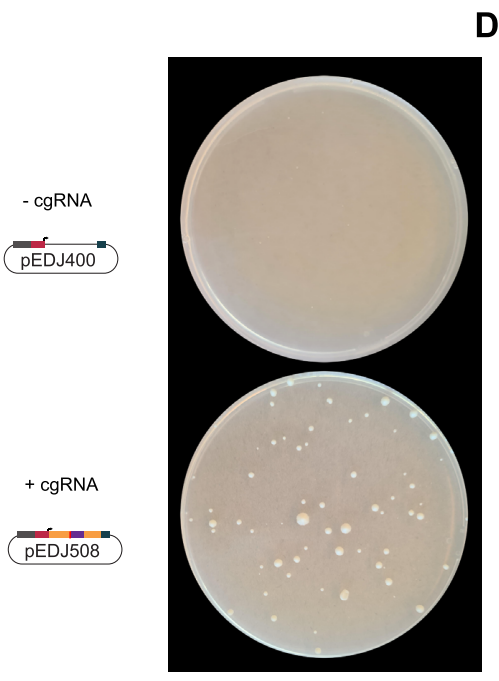

D

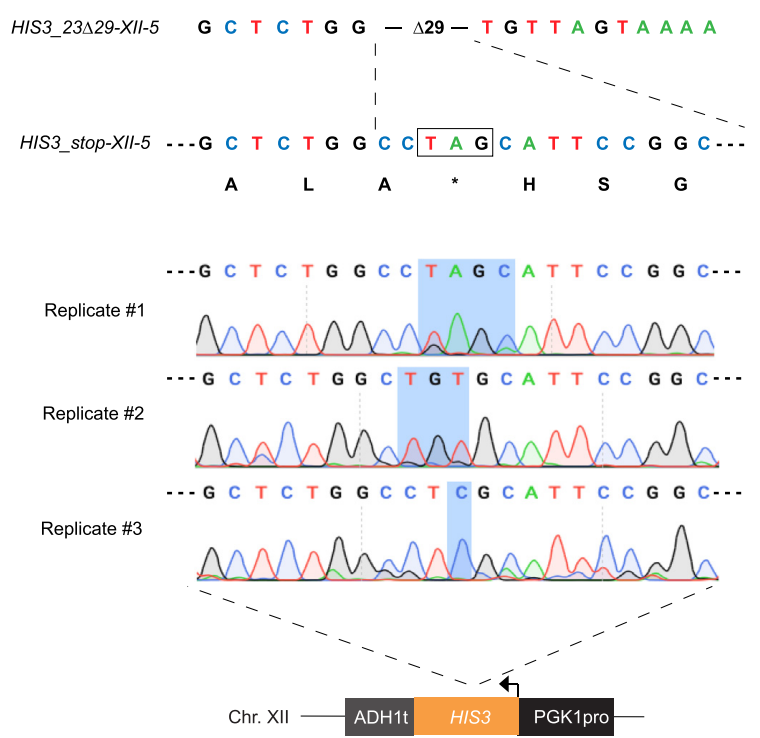

Figure 4. Directed evolution by cgRNA-DNA repair displays targeted transitions and transversion. (A) Plasmid-based galactose-inducible Cas9 and T7RNAPF11L/T613A (epT7RNAP) were expressed with plasmid pEDJ508 (described in the main text). On system induction for 48 hrs in synthetic complete dropout media with galactose, $c g R N A \_H I S 3 \_s t o p$ expressed from pEDJ508 directs Cas9 to the genome-integrated single copy HIS3_23 $229-X I I-5$ cassette to induce DNA double-strand break and template DNA repair. The cgRNA may contain the engineered STOP codon sequence (red), or evolved permissive mutations (green) introduced with epT7RNAP. (B) Biological replicates were induced $(\mathrm{ON})$ with galactose dropout media for $48 \mathrm{~h}$ in $2 \mathrm{ml}$ volumes. 300 $\mu \mathrm{l}$ were plated for each replicate on five plates containing histidine dropout media with glucose to stop evolution (OFF). (C) Pictures of representative plates were taken $72 \mathrm{~h}$ after plating of cultures expressing pEDJ400 (-cgRNA, top) or pEDJ508 (+cgRNA, bottom). (D) Sequencing results from amplicon sequencing of $500 \mu \mathrm{l}$ of saturated liquid cultures (population level) expressing a repaired HIS3 23 $\Delta 29-X I I-5$ allele (as indicated schematically at the bottom). Corresponding amino acids are shown below HIS3_stop-XII-5, and TAG (STOP) is boxed and indicated by an asterisk (*). Chromatograms are given for biological replicates \#1-3, where blue shading spans the range of mutated bases. Colony scores and $\mathrm{OD}_{600}$ values are presented in Supplementary Table S1.

propagated in liquid non-inducing selective conditions (glucose, w/o histidine) for three days to score growth. Indeed, while cultures carrying plasmid pEDJ508 grew to saturation, replicate cultures carrying pEDJ400 did not grow (Figure 4C), and neither did we observe any colonies on selective plates from cultures without cgRNA expression (Supplementary Table $\mathrm{S} 1$ ). Moreover, from amplicon sequencing of the repaired target site (i.e. HIS3_23 $\Delta 29-X I I-5$ ) of saturated cultures with pEDJ508, we found various mutations that abolished the STOP codon. Here, the replicate cultures carried one to three mutations containing either $\mathrm{T}>\mathrm{G}$ and $\mathrm{C}>\mathrm{A}$, translating into $\mathrm{STOP}>\mathrm{E}$ and $\mathrm{H}>\mathrm{N}, \mathrm{CTA}>\mathrm{TGT}$ translating into $\mathrm{STOP}>\mathrm{V}$, or one distinct mutation $\mathrm{A}>\mathrm{C}$ leading to $S T O P>S$ (Figure 4D). To further substantiate the ability of the plasmid-based CRAIDE system to selectively target genomic loci of interest, we also sequenced plasmid pools (pEDJ508) from replicate cultures. Here, the pre-engineered STOP codon in $c g R N A \_H I S 3 \_s t o p$ was observed in all of the cultures (Supplementary Figure S5B). Importantly, reintroduction of identified STOP codon mutations from the repaired HIS3 23 $\Delta 29-X I I-5$ genomic locus into clean genetic background strains verified histidine prototrophy in all cases (Supplementary Figure S5C). Thus, from this parallelized directed evolution study, CRAIDE 
conferred both transitions, transversions, and combinations thereof.

Finally, based on colony numbers from growth under selective conditions (see Supplementary Table S1), the initial mutation rate was estimated to be $9.77 \times 10^{-6}$ per cell per generation, and the per-base mutation rate was determined to be $3.26 \times 10^{-6}$ by adjusting for the number of bases (3) that can give rise to a permissive codon after repair of HIS3 23 229 -XII-5 (for detailed explanation see Methods section Estimation of mutation frequency and rate).

Taken together, from the genotyping of gain-of-function mutants, any base (A, T, C or G) can be introduced into the cgRNA during transcription and further transferred into a targeted genomic sequence, thus establishing inducible directed evolution in vivo based on RNA-mediated genome editing, with a mutation rate of $3.26 \times 10^{-6}$ per base, being $>3000$-fold higher than native background mutation frequency (1). Importantly, no mutants appeared on selective plates or in liquid cultures without cgRNA expressed (Figure $4 \mathrm{C}$ and Supplementary Table S1).

\section{DISCUSSION}

This study demonstrates RNA-mediated and CRISPRguided in vivo editing and mutagenesis at targeted genomic loci. To enable this, we first optimized orthogonal control of cgRNA expression using T7RNAP and insulated T7 promoters, and next demonstrated cgRNA-DNA repair on targeted genomic DSBs generated with Cas9. Extending from these results, by using an error-prone variant of T7RNAP for in vivo delivery of cgRNAs with random mutations, we enabled the first demonstration of directed evolution based on long evolving RNA donor templates into genomic contexts using both Cas9 and dCas9, and without the use of in vitro supplied and pre-programmed repair donors, as routinely adopted in directed evolution systems $(5-7,48)$.

However, engineering in vitro and in vivo directed evolution systems has experienced a lot of attention since their first demonstrations landmarked by Wright and Joyce, and Esvelt et al., respectively $(8,23)$. For this reason, pros and cons should be addressed when developing novel directed evolution techniques. Here, compared to other in vivo directed evolution systems in yeast $(14,15,17)$, limitations of the current version of CRAIDE exist and need consideration and further improvement for the system to be applicable for efficient in vivo directed evolution across multiple species. Indeed, with a mutation rate in the order of $3.26 \times 10^{-6}$ per base, RNA-mediated repair of genomic contexts using variant RNA donors as demonstrated in this study is still 2-3 orders of magnitude less efficient compared to state-of-the-art in vivo directed evolution methods for bacteria, yeast, and mammalian cells, like OrthoRep, ICE and TRACE $(11,14,16,17,49)$. This hampers the adoption of CRAIDE in its current design for evolution-guided and massively parallelized studies of complex genetic traits, such as metabolic pathway engineering, unless a highthroughput screening or selection method is available. Furthermore, even though RNA-mediated DNA repair has previously been reported in wild-type yeast $(29,40)$, in its current version, CRAIDE requires disruption of host
RNases for successful RNA-mediated repair of genomic DSBs. Such genetic prerequisites restrict the immediate portability of the system to genetically tractable hosts for which RNase H disruption does not confer lethality (50). However, for such cases, one mitigation strategy could involve conditional mutants to relieve potential lethality or long-term genotoxicity. Likewise, whereas $S$. cerevisiae has a highly proficient homologous recombination machinery for DNA repair, other eukaryotes, including mammals, are biased towards NHEJ to repair genomic DSBs (51) and may undermine, or at least limit, cgRNA-DNA repair efficiency, which is an essential requirement for CRAIDE to function. However, as reported for mammalian cells, designing physical proximity between targeted genomic loci and gRNA-appended donors can limit such false-positive events (7). To avoid generation of indel mixtures from using Cas9 for genome editing (6), our successful demonstration of CRAIDE for genome editing using dCas9 should be of relevance for in vivo directed evolution in hosts with NHEJbias for DSB repair. Also, homologous recombination can be further prompted to facilitate CRAIDE in new hosts by directly fusing HDR enhancing proteins to Cas9 $(52,53)$. Moreover, target genes can be engineered prior to CRAIDE to completely avoid screening for mutants that result from NHEJ, by removal of bases adjacent to PAM, which are then subsequently re-introduced by cgRNA-DNA repair as was performed in this study. As more findings on mechanisms governing RNA-DNA repair emerge, new strategies, such as fusing DNA polymerase $\zeta$ or other polymerases possibly involved in RNA-DNA repair (54), are relevant to pursue.

Acknowledging these limitations and considerations, CRAIDE is still a complementary tool expanding the scope of existing in vivo directed evolution systems $(14,15,17)$, and to the best of our knowledge the first to directly utilize erroneous RNA-templated DNA repair. Specifically, CRAIDE constitutes a versatile in vivo directed evolution system with tunability in terms of editing efficiency (e.g. cgRNA expression, length of donor), flexibility in terms of genomic target loci (Cas9-directed genic and intergenic regions), and mutational landscape determined by T7RNAP fidelity (any base, transversions and transitions), with a $>100$ bp editing window.

Interestingly, dCas9 also enabled targeted mutagenesis in vivo with cgRNA delivered from epT7RNAP (Supplementary Figure S4), and we speculate that two underlying mechanisms can account for this observation. First, DSBs can in fact occur from replication fork stalling and collapse posed by obstacles during replication (55), such as dCas9:cgRNA in complex with DNA. Nascent strand synthesis on open ends of single stranded DNA annealing to homologous mutant cgRNA then follows before break-induced replication or merge with passive replication to repair the DSB.

However, another scenario involving a DSB-independent mechanism cannot be ruled out, opening up the possibility that CRAIDE eventually could be applied in combination with complementary technologies, and in other model organisms, which are less proficient for homologous recombination. Such a mechanism may work through strand displacement and nascent strand synthesis using mutant 
cgRNA as template during replication fork stalling without collapse, prior to replication restart (55).

Lastly, it should be mentioned that during the preparation of this study, three novel DNA-templated genome editing technologies were reported; prime editor, TRACE and T7-DIVA $(7,11,16)$. Here, prime editor demonstrated RNA-mediated genome engineering using in vitro-edited donor-amended gRNAs (prime editing gRNAs) (7), while TRACE and T7-DIVA demonstrated that T7RNAP fused to base editors could be applied for continuous in vivo mutagenesis of target genes controlled by genomically integrated T7 promoters $(11,16)$. Individually, these new technologies enable $>10^{-4}$ mutations per base in engineered T7prodriven open reading frames sized up to $2 \mathrm{~kb}$, and nucleasedeficient integration of mutant bases in a prime editor window of approximately 30 bases $(7,11,16)$. In the future, we envision that the in vivo variant donor delivery and editing window size of CRAIDE together with the high editing efficiencies of these technologies could present appealing mergers for development of efficient in vivo continuous evolution in broad genomic contexts, as well as providing a tool for more foundational basic research on RNA-mediated evolution.

\section{SUPPLEMENTARY DATA}

Supplementary Data are available at NAR Online.

\section{ACKNOWLEDGEMENTS}

This work was supported by the Novo Nordisk Foundation. Author contributions: E.D.J., T.J., J.D.K. and M.K.J. conceived this project. E.D.J., T.J., L.E.P. and M.K.J. designed all of the experiments. E.D.J., M.L. and T.J. performed all growth and evolution assays. E.D.J. and M.L constructed all strains and plasmids. E.D.J, T.J., L.E.P. and M.K.J. analyzed the data. E.D.J. and M.K.J. wrote the paper.

\section{FUNDING}

Novo Nordisk Fonden. Funding for open access charge: Novo Nordisk Foundation.

Conflict of interest statement. JDK has a financial interest in Amyris, Lygos, Demetrix, Constructive Biology, Maple Bio, and Napigen.

\section{REFERENCES}

1. Lynch,M. (2010) Evolution of the mutation rate. Trends Genet., 26, 345-352.

2. Badran,A.H. and Liu,D.R. (2015) Development of potent in vivo mutagenesis plasmids with broad mutational spectra. Nat. Commun., 6, 8425 .

3. Simon,A.J., d'Oelsnitz,S. and Ellington,A.D. (2019) Synthetic evolution. Nat. Biotechnol., 37, 730-743.

4. Wang,H.H., Isaacs,F.J., Carr,P.A., Sun,Z.Z., Xu,G., Forest,C.R. and Church,G.M. (2009) Programming cells by multiplex genome engineering and accelerated evolution. Nature, 460, 894-898.

5. Sharon,E., Chen,S.-A.A., Khosla,N.M., Smith,J.D., Pritchard,J.K. and Fraser,H.B. (2018) Functional genetic variants revealed by massively parallel precise genome editing. Cell, 175, 544-557.

6. Roy,K.R., Smith,J.D., Vonesch,S.C., Lin,G., Tu,C.S., Lederer,A.R. Chu,A., Suresh,S., Nguyen,M., Horecka,J. et al. (2018) Multiplexed precision genome editing with trackable genomic barcodes in yeast. Nat. Biotechnol., 36, 512-520.
7. Anzalone,A.V., Randolph,P.B., Davis,J.R., Sousa,A.A., Koblan,L.W., Levy,J.M., Chen,P.J., Wilson,C., Newby,G.A., Raguram,A. et al. (2019) Search-and-replace genome editing without double-strand breaks or donor DNA. Nature, 576, 149-157.

8. Esvelt,K.M., Carlson,J.C. and Liu,D.R. (2011) A system for the continuous directed evolution of biomolecules. Nature, 472, 499-503.

9. Halperin,S.O., Tou,C.J., Wong,E.B., Modavi,C., Schaffer,D.V. and Dueber,J.E. (2018) CRISPR-guided DNA polymerases enable diversification of all nucleotides in a tunable window. Nature, $\mathbf{5 6 0}$, 248-252.

10. Moore,C.L., Papa,L.J. 3rd and Shoulders,M.D. (2018) A processive protein chimera introduces mutations across defined DNA regions in vivo. J. Am. Chem. Soc., 140, 11560-11564.

11. Álvarez,B., Mencía,M., de Lorenzo,V. and Fernández,L.Á. (2020) In vivo diversification of target genomic sites using processive base deaminase fusions blocked by dCas9. Nat. Commun., 11, 6436.

12. Findlay,G.M., Boyle,E.A., Hause,R.J., Klein,J.C. and Shendure,J. (2014) Saturation editing of genomic regions by multiplex homology-directed repair. Nature, 513, 120-123.

13. Ravikumar,A., Arrieta,A. and Liu,C.C. (2014) An orthogonal DNA replication system in yeast. Nat. Chem. Biol., 10, 175-177.

14. Crook,N., Abatemarco,J., Sun,J., Wagner,J.M., Schmitz,A. and Alper,H.S. (2016) In vivo continuous evolution of genes and pathways in yeast. Nat. Commun., 7, 13051.

15. Finney-Manchester,S.P. and Maheshri,N. (2013) Harnessing mutagenic homologous recombination for targeted mutagenesis in vivo by TaGTEAM. Nucleic. Acids. Res., 41, e99.

16. Chen,H., Liu,S., Padula,S., Lesman,D., Griswold,K., Lin,A., Zhao,T., Marshall,J.L. and Chen,F. (2019) Efficient, continuous mutagenesis in human cells using a pseudo-random DNA editor. Nat. Biotechnol., 38, 165-168.

17. Ravikumar,A., Arzumanyan,G.A., Obadi,M.K.A., Javanpour,A.A. and Liu,C.C. (2018) Scalable, continuous evolution of genes at mutation rates above genomic error thresholds. Cell, 175, 1946-1957.

18. Morrison,M.S., Podracky,C.J. and Liu,D.R. (2020) The developing toolkit of continuous directed evolution. Nat. Chem. Biol., 16, 610-619.

19. Liu,C.C., Jewett,M.C., Chin,J.W. and Voigt,C.A. (2018) Toward an orthogonal central dogma. Nat. Chem. Biol., 14, 103-106.

20. Tizei,P.A.G., Csibra,E., Torres,L. and Pinheiro,V.B. (2016) Selection platforms for directed evolution in synthetic biology. Biochem. Soc. Trans. , 44, 1165-1175.

21. Sanjuán,R., Nebot,M.R., Chirico,N., Mansky,L.M. and Belshaw,R. (2010) Viral mutation rates. J. Virol., 84, 9733-9748.

22. Simon,A.J., Morrow,B.R. and Ellington,A.D. (2018) Retroelement-based genome editing and evolution. ACS Synth. Biol., 7, 2600-2611.

23. Wright,M.C. and Joyce,G.F. (1997) Continuous in vitro evolution of catalytic function. Science, 276, 614-617.

24. Storici,F., Bebenek,K., Kunkel,T.A., Gordenin,D.A. and Resnick,M.A. (2007) RNA-templated DNA repair. Nature, 447, 338-341.

25. Shen,Y., Nandi,P., Taylor,M.B., Stuckey,S., Bhadsavle,H.P., Weiss,B. and Storici,F. (2011) RNA-driven genetic changes in bacteria and in human cells. Mutat. Res., 717, 91-98.

26. Mazina,O.M., Keskin,H., Hanamshet,K., Storici,F. and Mazin,A.V. (2017) Rad52 inverse strand exchange drives RNA-iemplated DNA double-strand break repair. Mol. Cell, 67, 19-29.

27. McDevitt,S., Rusanov,T., Kent,T., Chandramouly,G. and Pomerantz,R.T. (2018) How RNA transcripts coordinate DNA recombination and repair. Nat. Commun., 9, 1091.

28. Keskin,H., Shen,Y., Huang,F., Patel,M., Yang,T., Ashley,K., Mazin,A.V. and Storici,F. (2014) Transcript-RNA-templated DNA recombination and repair. Nature, 515, 436-439.

29. Keskin,H., Meers,C. and Storici,F. (2016) Transcript RNA supports precise repair of its own DNA gene. RNA Biol., 13, 157-165.

30. Gasiunas,G., Barrangou,R., Horvath,P. and Siksnys,V. (2012) Cas9-crRNA ribonucleoprotein complex mediates specific DNA cleavage for adaptive immunity in bacteria. Proc. Natl. Acad. Sci. U.S.A., 109, E2579-E2586.

31. Jinek,M., Chylinski,K., Fonfara,I., Hauer,M., Doudna,J.A. and Charpentier,E. (2012) A programmable dual-RNA-guided DNA endonuclease in adaptive bacterial immunity. Science, 337, 816-821. 
32. Cong,L., Ran,F.A., Cox,D., Lin,S., Barretto,R. and Habib,N. (2013) Multiplex genome engineering using CRISPR/Cas systems. Science, 339, 819-823.

33. DiCarlo,J.E., Norville,J.E., Mali,P., Rios,X., Aach,J. and Church,G.M. (2013) Genome engineering in Saccharomyces cerevisiae using CRISPR-Cas systems. Nucleic. Acids. Res., 41, 4336-4343.

34. Reid-Bayliss,K.S. and Loeb,L.A. (2017) Accurate RNA consensus sequencing for high-fidelity detection of transcriptional mutagenesis-induced epimutations. Proc. Natl. Acad. Sci. U.S.A., 114, 9415-9420.

35. Chen,W., Tabor,S. and Struhl,K. (1987) Distinguishing between mechanisms of eukaryotic transcriptional activation with bacteriophage T7 RNA polymerase. Cell, 50, 1047-1055.

36. Morse,N.J., Wagner,J.M., Reed,K.B., Gopal,M.R., Lauffer,L.H. and Alper,H.S. (2018) T7 polymerase expression of guide RNAs in vivo allows exportable CRISPR-Cas9 editing in multiple yeast hosts. ACS Synth. Biol., 7, 1075-1084.

37. Dower,K. and Rosbash,M. (2002) T7 RNA polymerase-directed transcripts are processed in yeast and link 3' end formation to mRNA nuclear export. $R N A, \mathbf{8}, 686-697$.

38. Brakmann,S. and Grzeszik,S. (2001) An error-prone T7 RNA polymerase mutant generated by directed evolution. ChemBioChem, 2, 212-219.

39. Bao,Z., Xiao,H., Liang,J., Zhang,L., Xiong,X., Sun,N., Si,T. and Zhao,H. (2015) Homology-integrated CRISPR-Cas (HI-CRISPR) system for one-step multigene disruption in Saccharomyces cerevisiae. ACS Synth. Biol., 4, 585-594.

40. Keskin,H., Shen,Y., Huang,F., Patel,M., Yang,T., Ashley,K., Mazin,A.V. and Storici,F. (2014) Transcript-RNA-templated DNA recombination and repair. Nature, 515, 436-439.

41. Derr,L.K., Strathern,J.N. and Garfinkel,D.J. (1991) RNA-mediated recombination in S. cerevisiae. Cell, 67, 355-364.

42. McDevitt,S., Rusanov,T., Kent,T., Chandramouly,G. and Pomerantz,R.T. (2018) How RNA transcripts coordinate DNA recombination and repair. Nat. Commun., 9, 1091.

43. Storici,F., Durham,C.L., Gordenin,D.A. and Resnick,M.A. (2003) Chromosomal site-specific double-strand breaks are efficiently targeted for repair by oligonucleotides in yeast. Proc. Natl. Acad. Sci. U.S.A., 100, 14994-14999.

44. Qi,L.S., Larson,M.H., Gilbert,L.A., Doudna,J.A., Weissman,J.S., Arkin,A.P. and Lim,W.A. (2013) Repurposing CRISPR as an RNA-guided platform for sequence-specific control of gene expression. Cell, 152, 1173-1183.

45. Hsu,P.D., Scott,D.A., Weinstein,J.A., Ran,F.A., Konermann,S., Agarwala,V., Li,Y., Fine,E.J., Wu,X., Shalem,O. et al. (2013) DNA targeting specificity of RNA-guided Cas9 nucleases. Nat. Biotechnol. 31, 827-832.

46. Richardson,C.D., Ray,G.J., DeWitt,M.A., Curie,G.L. and Corn,J.E. (2016) Enhancing homology-directed genome editing by catalytically active and inactive CRISPR-Cas9 using asymmetric donor DNA. Nat. Biotechnol., 34, 339-344.

47. Laughery,M.F., Mayes,H.C., Pedroza,I.K. and Wyrick,J.J. (2019) $\mathrm{R}$-loop formation by dCas9 is mutagenic in Saccharomyces cerevisiae. Nucleic. Acids. Res., 47, 2389-2401.

48. Jakočiūnas,T., Pedersen,L.E., Lis,A.V., Jensen,M.K. and Keasling,J.D. (2018) CasPER, a method for directed evolution in genomic contexts using mutagenesis and CRISPR/Cas9. Metab. Eng., 48, 288-296.

49. Hess,G.T., Frésard,L., Han,K., Lee,C.H., Li,A., Cimprich,K.A., Montgomery,S.B. and Bassik,M.C. (2016) Directed evolution using
dCas9-targeted somatic hypermutation in mammalian cells. Nat. Methods, 13, 1036-1042.

50. Cerritelli,S.M., Frolova,E.G., Feng,C., Grinberg,A., Love,P.E. and Crouch,R.J. (2003) Failure to produce mitochondrial DNA results in embryonic lethality in Rnaseh1 null mice. Mol. Cell, 11, 807-815.

51. Emerson,C.H. and Bertuch,A.A. (2016) Consider the workhorse: nonhomologous end-joining in budding yeast. Biochem. Cell Biol., 94, 396-406.

52. Song,J., Yang,D., Xu,J., Zhu,T., Chen,Y.E. and Zhang,J. (2016) RS-1 enhances CRISPR/Cas9- and TALEN-mediated knock-in efficiency. Nat. Commun., 7, 10548

53. Shao,S., Ren,C., Liu,Z., Bai,Y., Chen,Z., Wei,Z., Wang,X., Zhang,Z. and Xu,K. (2017) Enhancing CRISPR/Cas9-mediated homology-directed repair in mammalian cells by expressing Saccharomyces cerevisiae Rad52. Int. J. Biochem. Cell Biol., 92, 43-52.

54. Meers,C., Keskin,H., Banyai,G., Mazina,O., Yang,T., Gombolay,A.L., Mukherjee,K., Kaparos,E.I., Newnam,G., Mazin,A. et al. (2020) Genetic characterization of three distinct mechanisms supporting RNA-driven DNA repair and modification reveals major role of DNA polymerase $\zeta$. Mol. Cell, 79, 1037-1050.

55. Aguilera,A. and Gómez-González,B. (2008) Genome instability: a mechanistic view of its causes and consequences. Nat. Rev. Genet., 9 , 204-217.

56. Bitinaite,J., Rubino,M., Varma,K.H., Schildkraut,I., Vaisvila,R. and Vaiskunaite,R. (2007) USER ${ }^{\mathrm{TM}}$ friendly DNA engineering and cloning method by uracil excision. Nucleic. Acids. Res., 35, 1992-2002.

57. Jensen,N.B., Strucko,T., Kildegaard,K.R., David,F., Maury,J., Mortensen,U.H., Forster,J., Nielsen,J. and Borodina,I. (2014) EasyClone: method for iterative chromosomal integration of multiple genes in Saccharomyces cerevisiae. FEMS Yeast Res., 14, 238-248.

58. Jakočiūnas,T., Bonde,I., Herrgård,M., Harrison,S.J., Kristensen,M., Pedersen,L.E., Jensen,M.K. and Keasling,J.D. (2015) Multiplex metabolic pathway engineering using CRISPR/Cas9 in Saccharomyces cerevisiae. Metab. Eng., 28, 213-222.

59. Partow,S., Siewers,V., Biørn,S., Nielsen,J. and Maury,J. (2010) Characterization of different promoters for designing a new expression vector in Saccharomyces cerevisiae. Yeast, 27, 955-964.

60. Jessop-Fabre,M.M., Jakočiūnas,T., Stovicek,V., Dai,Z., Jensen,M.K., Keasling,J.D. and Borodina,I. (2016) EasyClone-MarkerFree: a vector toolkit for marker-less integration of genes into Saccharomyces cerevisiae via CRISPR-Cas9. Biotechnol. J., 11, 1110-1117.

61. Jensen,E.D., Ferreira,R., Jakočiūnas,T., Arsovska,D., Zhang,J., Ding,L., Smith,J.D., David,F., Nielsen,J., Jensen,M.K. et al. (2017) Transcriptional reprogramming in yeast using dCas9 and combinatorial gRNA strategies. Microb. Cell Fact., 16, 46.

62. Mairhofer,J., Wittwer,A., Cserjan-Puschmann,M. and Striedner,G. (2015) Preventing T7 RNA polymerase read-through transcription - a synthetic termination signal capable of improving bioprocess stability. ACS Synth. Biol., 4, 265-273.

63. Gillet-Markowska,A., Louvel,G. and Fischer,G. (2015) bz-rates: a web tool to estimate mutation rates from fluctuation analysis. $G 3, \mathbf{5}$, 2323-2327.

64. Zheng,Q. (2017) rSalvador: an R package for the fluctuation experiment. G3, 7, 3849-3856.

65. Verduyn,C., Postma,E., Scheffers,W.A. and Van Dijken,J.P. (1992) Effect of benzoic acid on metabolic fluxes in yeasts: a continuous-culture study on the regulation of respiration and alcoholic fermentation. Yeast, 8, 501-517. 